\title{
Research Paper \\ Effect of 12-Week Neck, Core, and Combined Stabilization Exercises on the Pain and Disability of Elderly Patients With Chronic Non-specific Neck Pain: A Clinical Trial
}

\author{
Farhad Azadi ${ }^{1}$, Reza Nabi Amjad ${ }^{2}$, Hossein Marioryad ${ }^{3}$, Monir Alimohammadi ${ }^{4}$, Alireza Karimpour Vazifehkhorani ${ }^{5}$, ${ }^{*}$ Mohsen \\ Poursadeghiyan $^{6}$
}

1. Iranian Research Center on Aging, University of Social Welfare and Rehabilitation Sciences, Tehran, Iran

2. Non-communicable Diseases Research Center, Alborz University of Medical Sciences, Karaj, Iran.

3. Social Determinants of Health Research Center, Yasuj University of Medical Sciences, Yasuj, Iran.

4. Health Management and Economics Research Center, Iran University of Medical Sciences, Tehran, Iran.

5. Department of Psychology, Faculty of Education and Psychology, University of Tabriz, Tabriz, Iran.

6. Health in Emergencies \& Disasters Research Center, University of Social Welfare and Rehabilitation Sciences, Tehran, Iran.

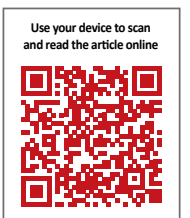

eftat on Azadi F, Nabi Amjad R, Marioryad H, Alimohammadi M, Karimpour Vazifehkhorani A, Poursadeghiyan M. [Effect of 12-Week Neck, Core, and Combined Stabilization Exercises on the Pain and Disability of Elderly Patients With Chronic Non-specific Neck Pain: AClinical Trial (Persian)]. Salmand: Iranian Journal of Ageing. 2019; 13(Special Issue):614-625. https://doi.org/10.32598/SIJA.13.Special-Issue.614

https://doi.org/10.32598/SIJA.13.Special-Issue.614

(ㄷ) 03

Received: 17 Jun 2018 Accepted: 13 Nov 2018 Available Online: 10 Mar 2019

Key words:

Neck stabilization exercises, Core stabilization exercises, Combined stabilization exercises, Non-specific chronic neck pain

\section{A B STRACT}

Objectives To investigate the effect of 12 weeks of neck stabilization, core stabilization, and combined stabilization exercises on pain and disability among elderly people in Tehran City, Iran.

Methods \& Materials This study was a 12 weeks open-label clinical trial. A total of 18 elderly patients with chronic neck pain were randomly assigned into three groups: neck stabilization training (6 people), core stabilization training ( 6 people), and combined stabilization training ( 6 people). The severity of neck pain and disability before the beginning of the training, 8 weeks after training and one week after the completion of the exercises were measured using the Visual Analog Scale (VAS), Neck Disability Index (NDI) and Neck Pain and Disability Scale (NPDI). To investigate the effect of time, repeated measure analysis of variance was used to analyze the data in SPSS version 21.

Results The Mean $\pm S D$ scores of pain before and after neck stabilization treatment were respectively $6.08 \pm 0.58,4.83 \pm 0.52$ for VAS and $49.17 \pm 2.86$ and $39.17 \pm 2.79$ for NDI; and $56.4 \pm 2.11$ and $50.0 \pm 1.64$ for NPDI; those differences between pairs were significant. The Mean \pm SD scores of pain before and after core stabilization treatment were respectively, $6.00 \pm 0.55,4.92 \pm 0.20$ for VAS; $49.67 \pm 1.86$ and $39.17 \pm 1.94$ for NDI; and 56.01 \pm 2.44 , and 48.92 \pm 1.16 for NPDI; those differences between pairs were significant. Also, the Mean $\pm S D$ scores of pain before and after combined stabilization treatment were respectively, $6.00 \pm 0.45,4.00 \pm 0.32$ for VAS; $49.83 \pm 2.23$ and $37.17 \pm 2.86$ for NDI; and $55.25 \pm 0.28$ and $47.51 \pm 1.44$ for NPDI; those differences between pairs were significant $(P<0.05)$. Among the underlying variables, gender was the only significant factor in pain relief in the elderly $(F=6.21, P=0.02)$, while other variables were not significant $(P>0.05)$.

Conclusion The findings of this study showed that 12 sessions of neck, core, and combined stabilization training in the neck region could improve the tolerance and pain of the elderly with nonspecific chronic neck pain.

\section{* Corresponding Author:}

Mohsen Poursadeghiyan, PhD.

Address: Health in Emergencies \& Disasters Research Center, University of Social Welfare and Rehabilitation Sciences, Tehran, Iran.

Tel: +98 (21) 22180160

E-mail: mo.poursadeghiyan@uswr.ac.ir 


\section{Extended Abstract}

\section{Objectives}

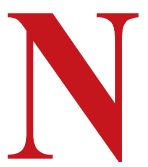

eck pain is one of the most common musculoskeletal disorders in modern societies. It is estimated that approximately $67 \%$ of people have experienced neck pain at least once in their life [1-4]. Neck pain is more prevalent in middle age and old age and can lead to severe disability in $5 \%$ of patients $[5,6]$. Increased prevalence of neck pain and its related disabilities along with increasing age and health care is an important challenge for the health care system $[7,8]$. Today, exercise therapy as an effective method for neck pain treatment has been accepted but is still considered as a major challenge [9]. In this regard, this study attempted to study the effect of neck stabilization, core stabilization, and combined stabilization exercises on the pain and disability of elderly patients with chronic Nonspecific Neck Pain (NSNP).

\section{Methods \& Materials}

This is an open-label trial conducted for 12 weeks on 18 elders with NSNP. They were selected using convenience sampling method and matched for gender and education. The inclusion criteria were aged between 65 and 80 years, with NSNP for 12 weeks and more, and with Visual Ana$\log$ Scale (VAS) score of 3. On the other hand, the exclusion criteria were a history of neck surgery, doing regular exercises, a history of a specific disease except neck pain, mental illness, cardiovascular disease, and neurological diseases, failure to complete treatment courses, simultaneous use of other therapies, and exacerbation of pain and disability by conducting training exercises. The participants were divided into three groups of neck stabilization $(n=6)$, core stabilization $(\mathrm{n}=6)$, and combined stabilization exercises $(n=6)$. Training sessions were conducted under the supervision of a physiotherapist every other day for 12 weeks, each session lasted 25-30 minutes. By using VAS, Neck Disability Index (NDI), and Neck Pain and Disability Scale (NPDS), the severity of neck pain and disability before the beginning of exercises was measured at the end of the 8th week and one week after the completion of the exercises.

\section{Results}

Of 18 participants, $39.9 \%$ were female and $60.1 \%$ male with a Mean \pm SD age of $68.28 \pm 4.61$ years. According to the results, neck stabilization exercises significantly reduced neck pain over time $(\mathrm{P}=0.008)$ where the Mean $\pm \mathrm{SD}$ pain VAS score reduced from $6.08 \pm 0.58$ at the beginning of the study to $4.83 \pm 0.52$ at 12 weeks after the treatment.
The pain NDI and NPDS mean scores also reduced about 10 and 6.39 units, respectively $(\mathrm{P}<0.001)$. Using core stabilization exercises, the pain VAS score reduced 1.08 unit $(\mathrm{P}=0.018)$, while pain NDI and NPDS scores reduced 10.5 and 7.09 units, respectively $(\mathrm{P}=0.000)$. Finally, the use of combined stabilization exercises, the pain VAS, NDI, and NPDS scores significantly reduced 2, 12.66, and 7.77 units, respectively $(\mathrm{P}<0.001)$.

The pain VAS, NDI and NPDS Mean \pm SD scores in core stabilization group before and after intervention were $6 \pm 0.55$ and $4.92 \pm 0.2 ; 49.1 \pm 67.86$ and $39.17 \pm 1.94$; $56.01 \pm 2.44$ and $48.92 \pm 1.16$, respectively $(\mathrm{P}<0.05)$. In combined stabilization group, these values were $6 \pm 0.45$ and $4 \pm 0.32 ; 49.2 \pm 83.23$ and $37.17 \pm 2.86$; and $55.25 \pm 0.28$ and 47.51 \pm 1.44 , respectively $(\mathrm{P}<0.05)$.

Comparing VAS and NDI scores, the difference in pain score between the pre-workout, 8 weeks and 12 weeks after the workout was significant by considering the effect of underlying factors and groups $(\mathrm{P}<0.001)$. The difference in pain reduction between the study groups by using NDI was significant $(\mathrm{P}=0.043)$. By using NDI, gender factor $(\mathrm{P}=0.02)$ had a significant effect on the elderly's pain reduction. By using NPDS, pain reduction was also significant over time $(\mathrm{P}<0.001)$. The difference in pain reduction between study groups was also significant $(\mathrm{P}=0.039)$.

\section{Conclusion}

Twelve weeks of neck stabilization, core stabilization, and combined stabilization exercises resulted in a significant improvement in the mean reduction in VAS, NDI and NPDS pain scores in the elderly with chronic NSNP. Among underlying factors, only the gender factor had a significant effect on their pain reduction. The findings also showed that the mean pain reduction in combined stabilization exercises was higher than in the neck and core stabilization exercises.

\section{Ethical Considerations}

\section{Compliance with ethical guidelines}

This study complied with ethical policy of University of Social Welfare and Rehabilitation Sciences (IR.USWR. REC.1396.156). This study was registered in Iranian Registry of Clinical Trials (IRCT20180412039280N2).

\section{Funding}

This study was financially supported by Iranian Research Center of Aging on University of Social Welfare and Rehabilitation Sciences (Grant number:1534). 
Authors' contributions

All authors contributed in designing, running, and writing all parts of the research.

\section{Conflict of interest}

The authors declared no conflict of interest.

\section{Acknowledgments}

Authors would like to thank all participants and Iranian Research Center on Aging of University of Social Welfare and Rehabilitation Sciences for their valuable cooperation. 


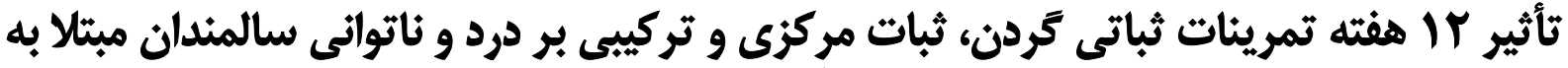

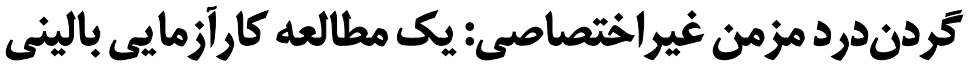

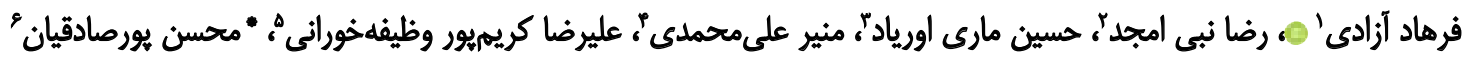

1- مركز تحقيقات سالمندى، دانشعاه علوم بهزيستى و توانبخشى، تهران، ايران.

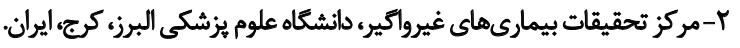

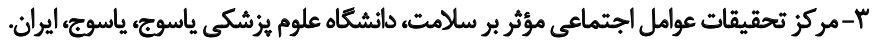

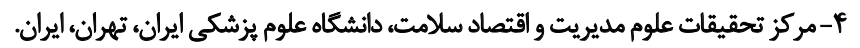

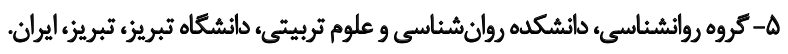

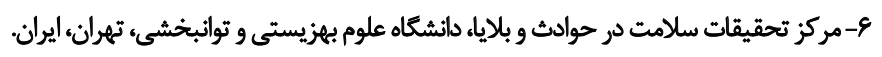

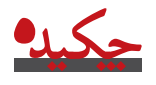

الهدافت اين تحقيق بررسى تأثير كا هفته تمريثات ثباتى كردن، ثبات مركزى و تركيبى بر درد و ناتوانى افراد مبتلا به كردندرد مزمن

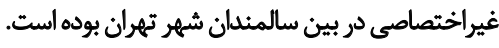

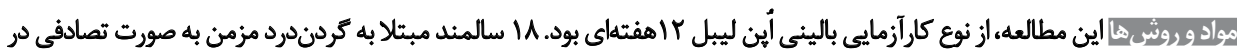

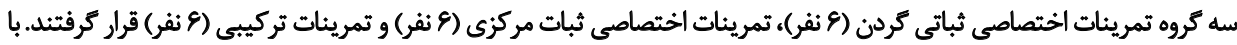

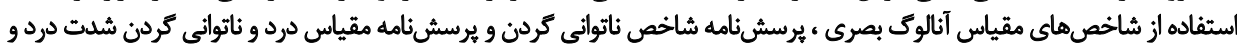

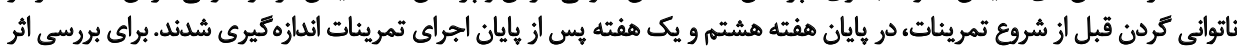

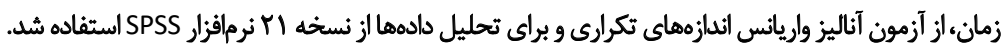

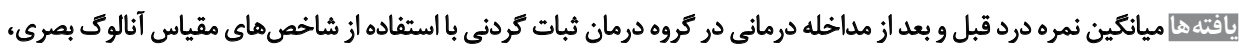

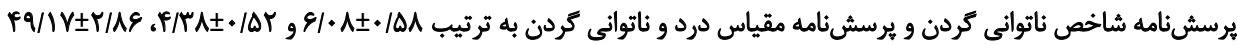
و و قبل و بعد از مداخله درمانى در كروه درمان ثبات مركزي به توتيب

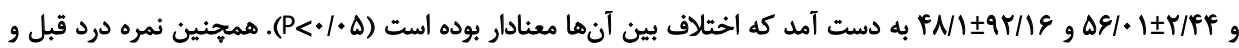

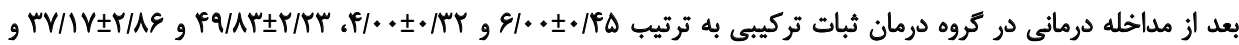

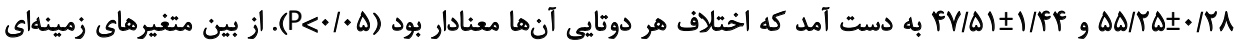

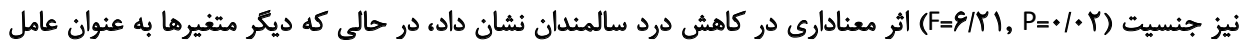

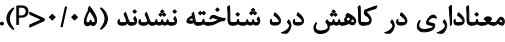

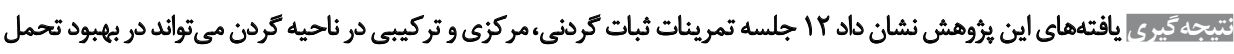

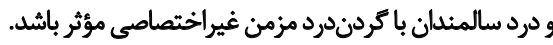

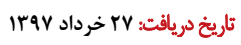

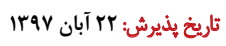
تاريخ انتشار: 19 اسفند
كليدوأرها:

تمرينات ثباتى تركن، تمرينات ثبات مركزى تئ،

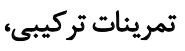

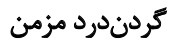

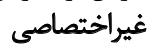

اسكلتى عضلانى شايع در جوامع امروزى است؛ به طورى كورى كه تقريباً

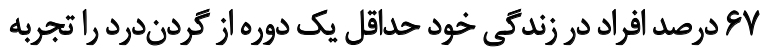

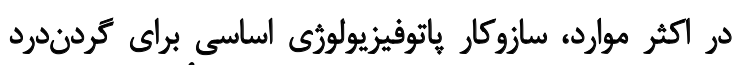

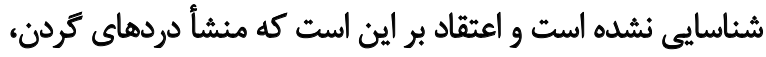

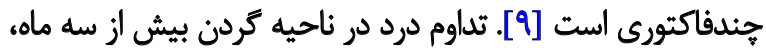

اختلالات اسكلتىعضلاتى به هركونه آسيب بافتى در سيستم

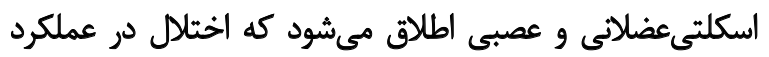

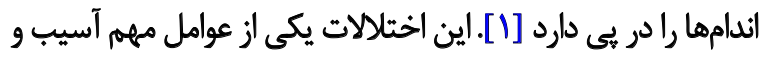

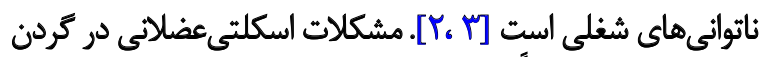

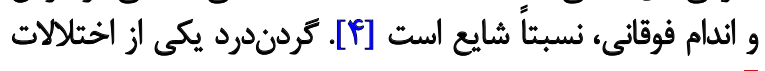

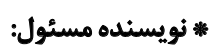

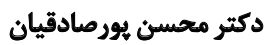

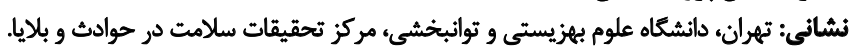

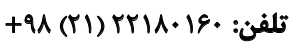
بست الكترونيكي: mo.poursadeghiyan@uswr.ac.ir 
عبارتند از: استفاده از يك يا جند نوع تمريندرمانى شامل تمرينات

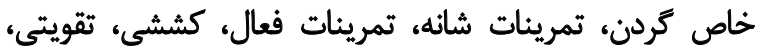

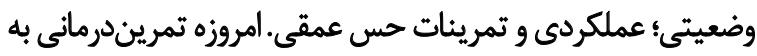

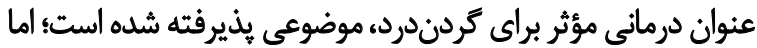

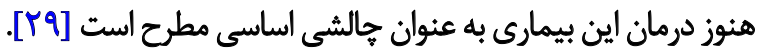
ثبات مركزى به عنوان كنترل حركتى وظرفيتي عضئ عضلانى ناحيه

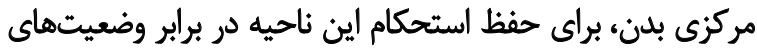

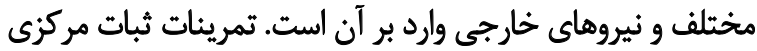

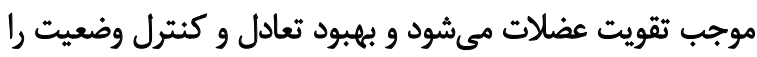

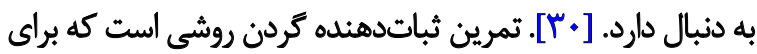

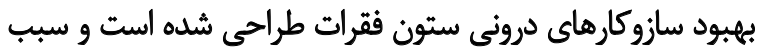

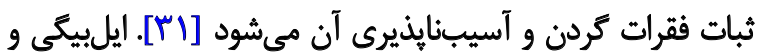

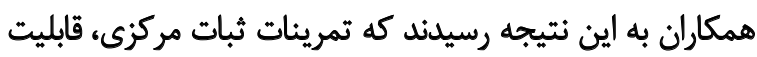

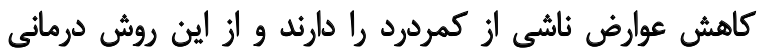

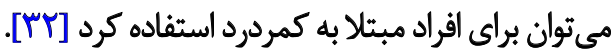
صفدرى و همكاران نشان دادند تمرينات ثباتدهنده در بردي

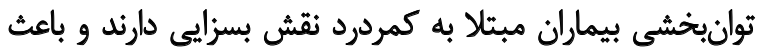

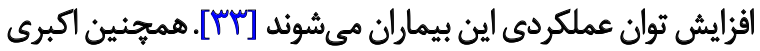

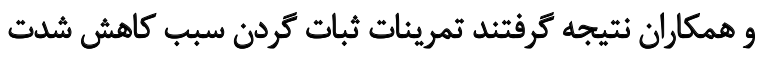

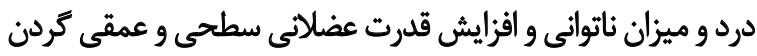

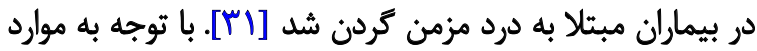

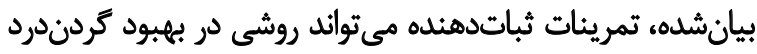

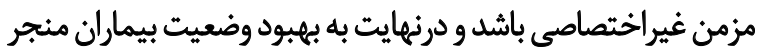

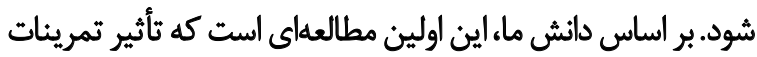

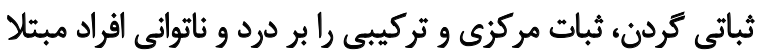

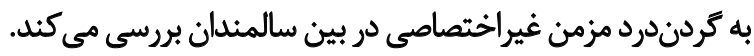

روش مطالعه

\section{بيمار ان و معيارهاي ورودو وخروج}

سالمندان با كردندرد مزمن غيراختصاصى وارد مطالعه شدند.

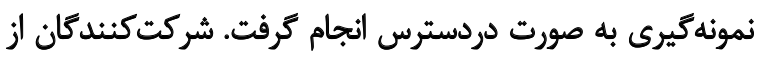

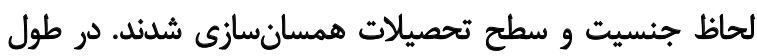

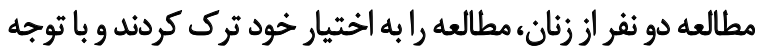

به نمونههاى دردسترس، به تعداد مردان دو نفر اضافه شد.

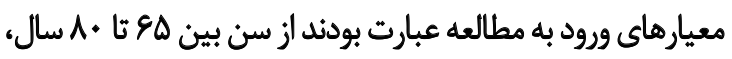

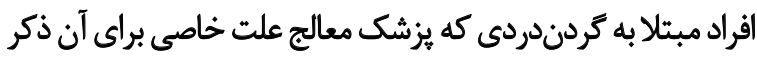

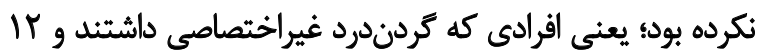

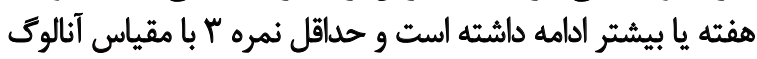

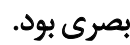

معيارهاى خروج از مطالعه عبارت بودند از سابقه جراحى در

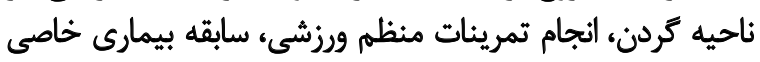

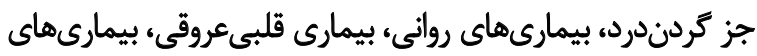

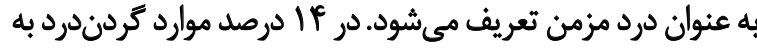

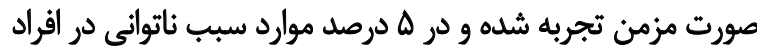

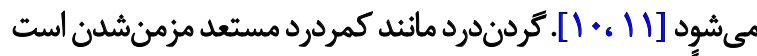

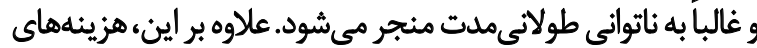

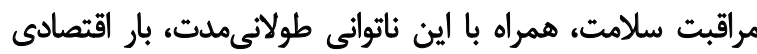

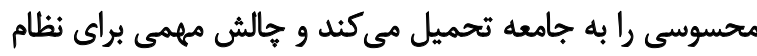

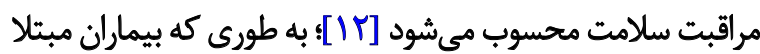

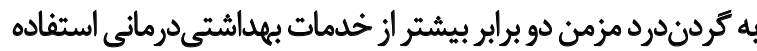

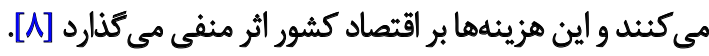

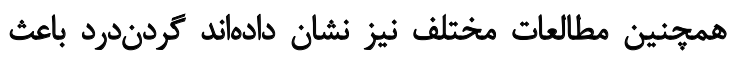

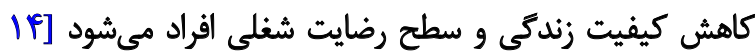

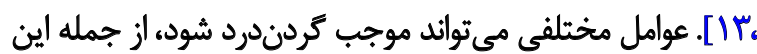

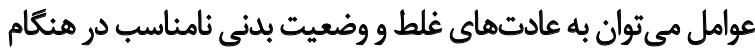

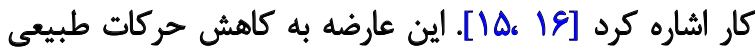

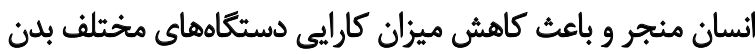

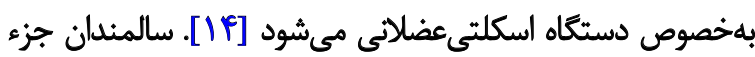

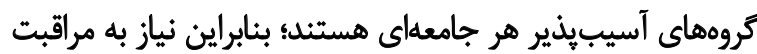

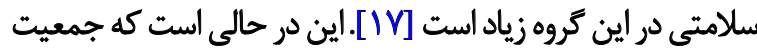

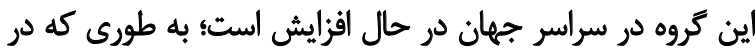

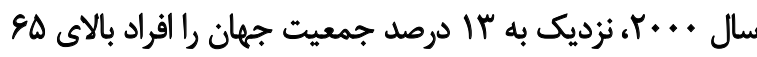

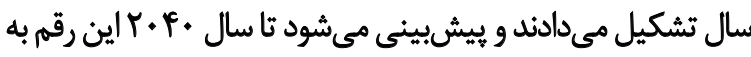

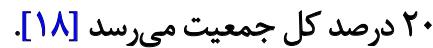

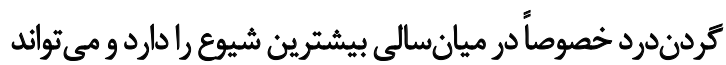

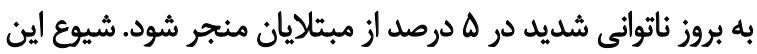

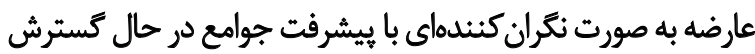

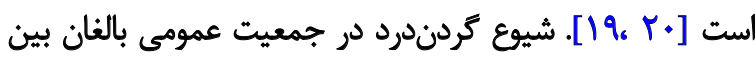

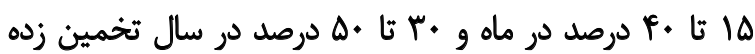

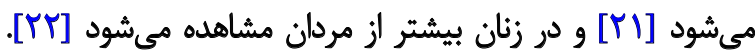

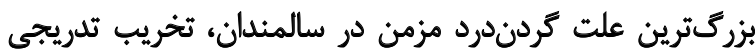

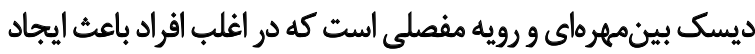

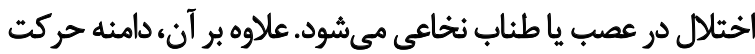

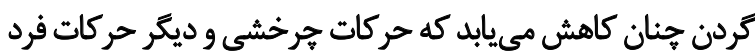

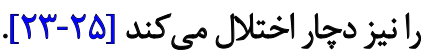

درمان اختلالات مزمن كردن متنوع است و شامل روشهاي

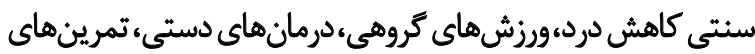

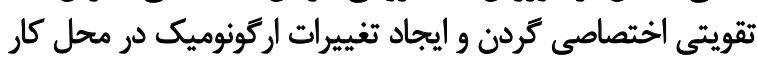

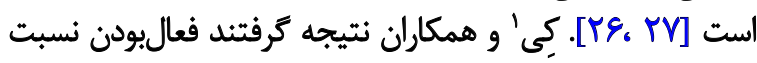

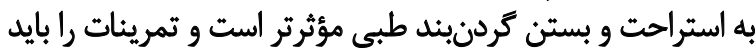

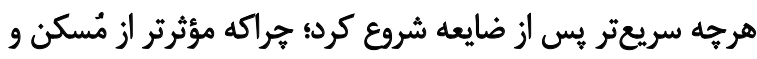

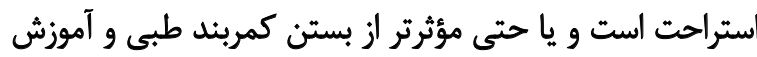

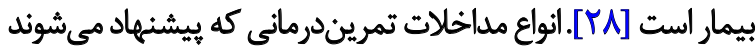

\section{Kay}


تبحليل آمارى

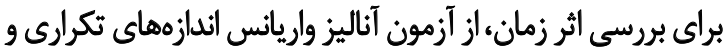

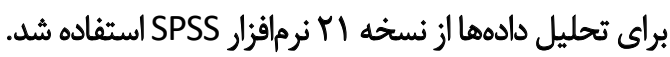

بافتلها

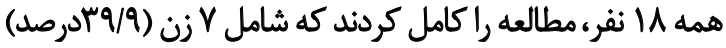

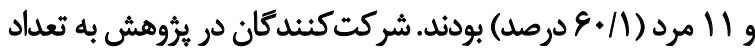

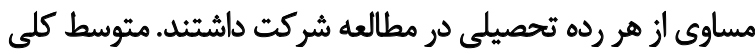

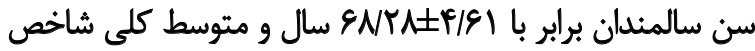

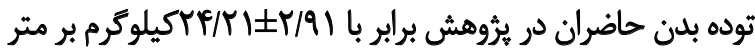

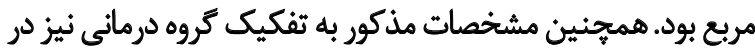
جدول شماره آمده است.

نتايج حاصل از ارزيابى هر سه روش اندازمكيرى درد نشان داد

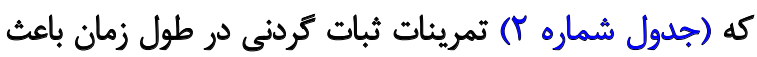

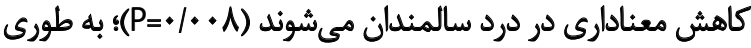

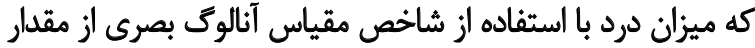

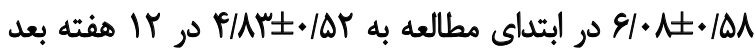

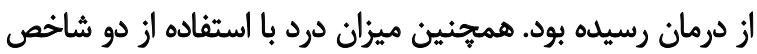

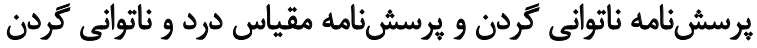

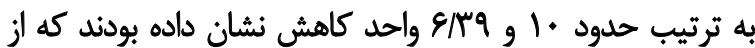

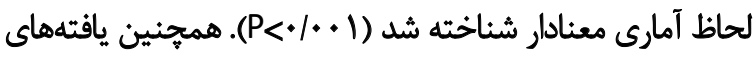

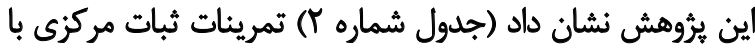

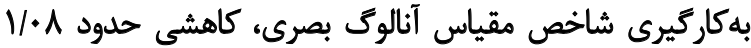

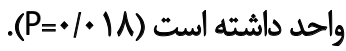

همجنان كه اين مقدار كاهش براى شاخص يرسش ثانامه ناتوانى

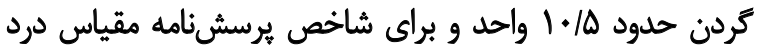

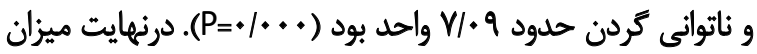

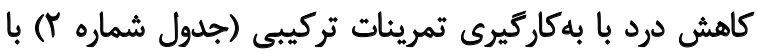

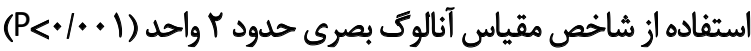

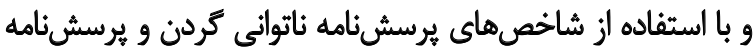

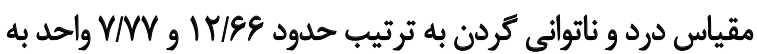

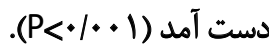

با واردكردن متغير تروه درمانى به عنوان مداخله درمانى

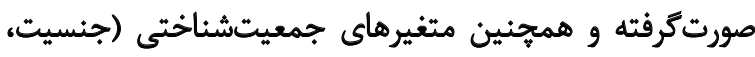

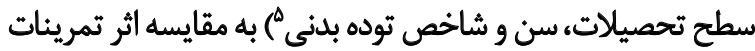

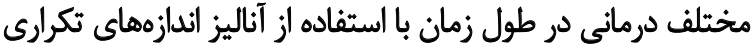

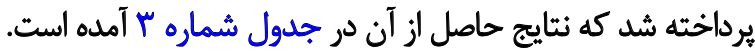

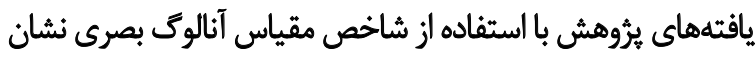

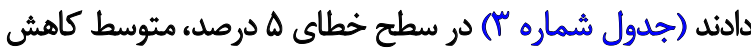
درد، در طول زمان، روندى معنادار داشته است؛ به به عبارت دئ ديكر
عصبى، تكميلنشدن دورههاى درمانى، استفاده همزمان از ديكر

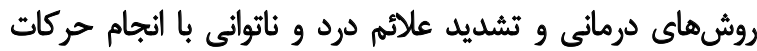

$$
\text { تمرينى. }
$$

$$
\text { طراحي مطالعه }
$$

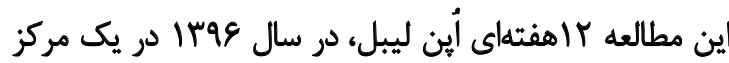

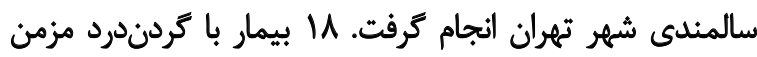

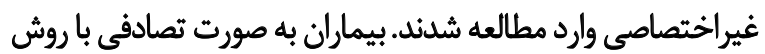

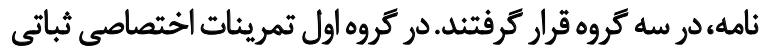

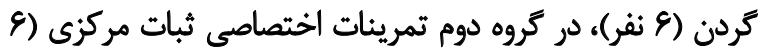

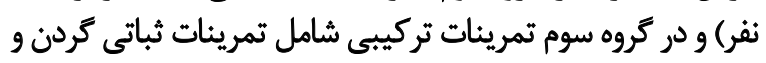

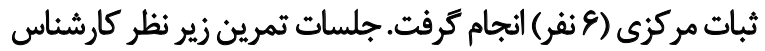

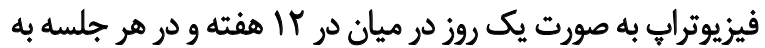

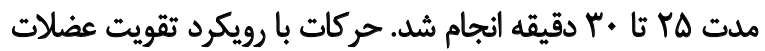

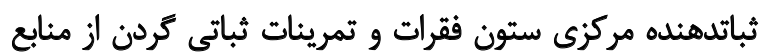

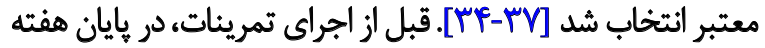

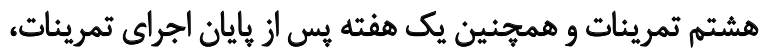

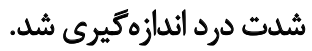

\section{اندازٔهيرى يبيامدها}

ابزارهاى جمعآورى اطلاعات شامل مقياس آنالوك بصرى؟

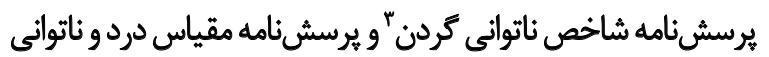

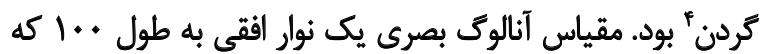

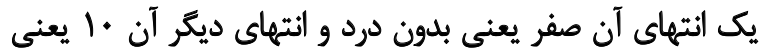

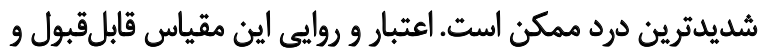

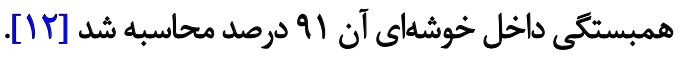
از يرسشنامه شاخص ناتوانى تردن براي سنجش ناتوانى در

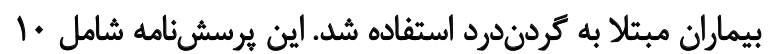

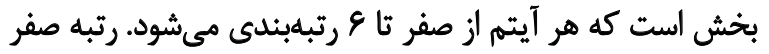

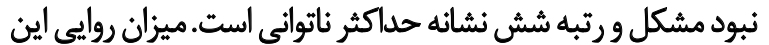

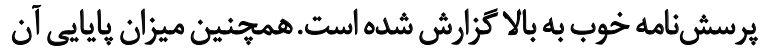

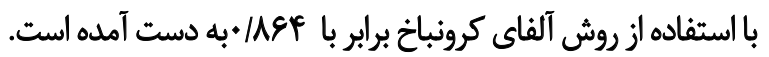

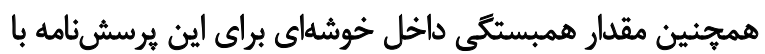

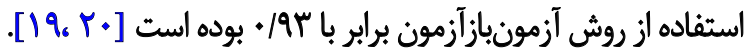

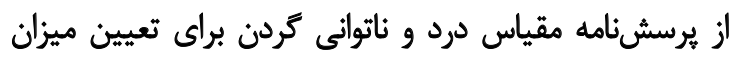

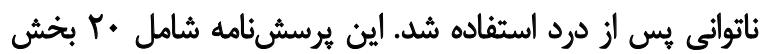

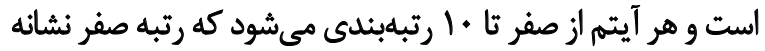

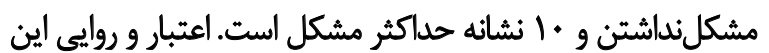

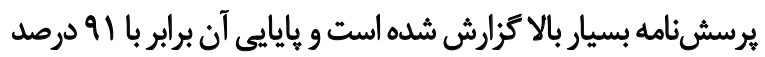

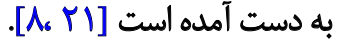

2. Visual Analogue Scale (VAS)

3. Neck Disability Index (NDI)

4. Neck Pain and Disability Scale (NPDS) 
اندازمكيرى درد (مقياس آنالوك بصرى، يرسشنامه شاخص ناتوانى

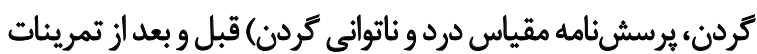

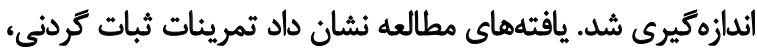

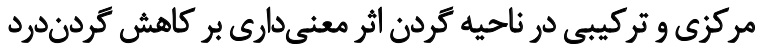

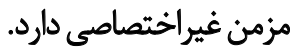
متوسط نمرات درد با هر سه مقياس كاهش معنادارى را نشان

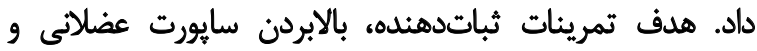

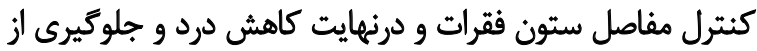

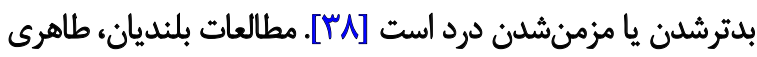

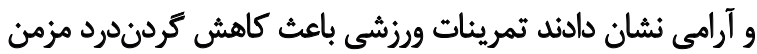

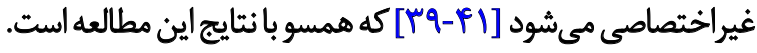
مطالعه لطافت كار و همكاران روى سالمندان مبتلا به كردندرد

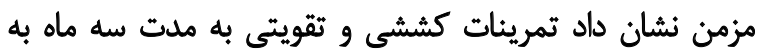

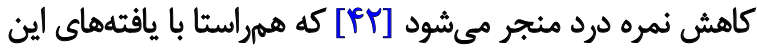

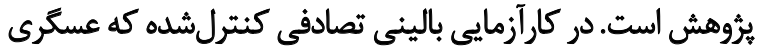

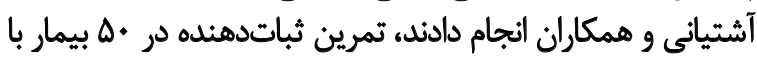

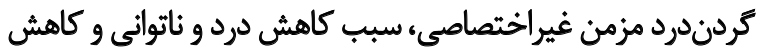

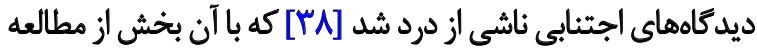

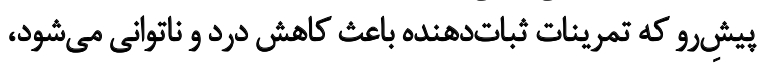

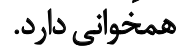

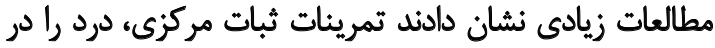

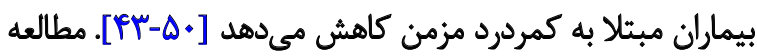

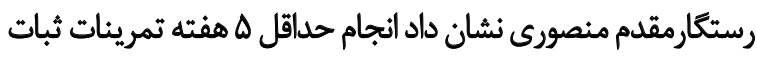

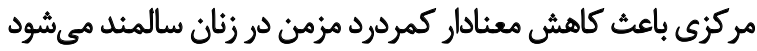

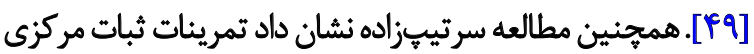

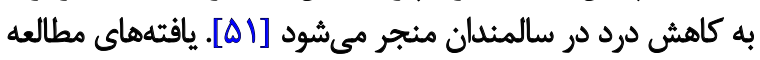

اختلاف نمره درد بين قبل از تمرين، \ هفته و I ا هفته بعد از

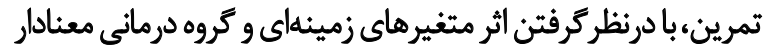

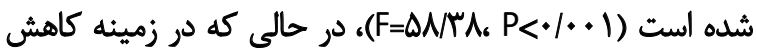

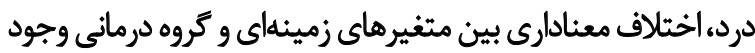

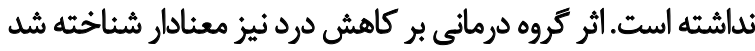

. $F=F / N q$, $P=\cdot / \cdot T Q)$

يافتهاى يرؤش در جدول شماره ب نشان داد با بهكاركيرى

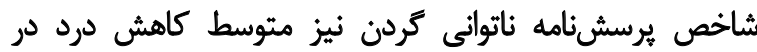

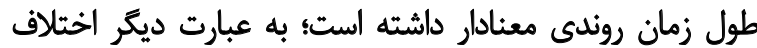

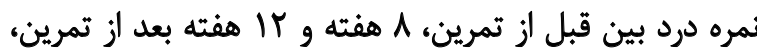

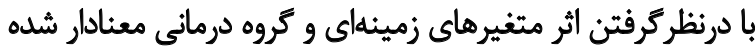

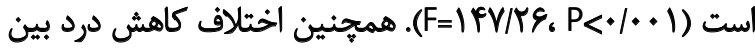

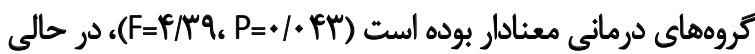

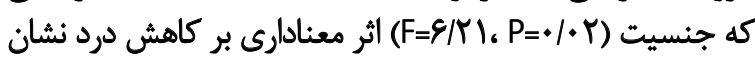

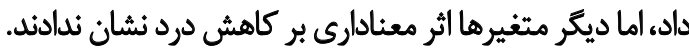

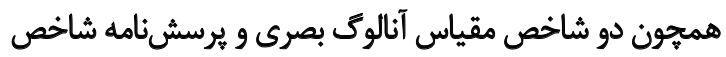

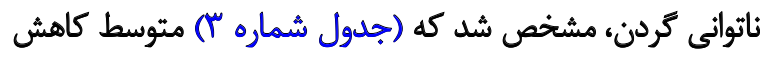

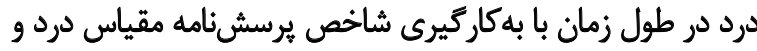

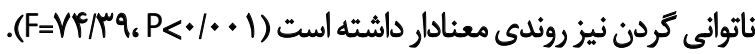

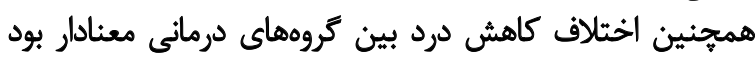

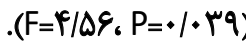

ثب

هدف از اين مطالعه، بررسى تأثير باتهفئهائ تمرينات ثباتي

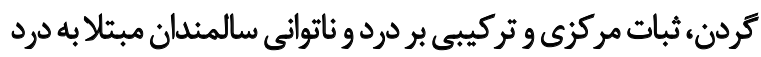

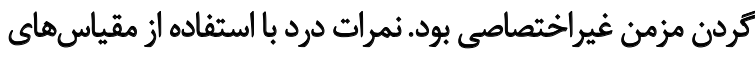

جدول ا. مشخصات شركت كنيندكان در برُوهش

\begin{tabular}{|c|c|c|c|}
\hline \multicolumn{3}{|c|}{ ميروه درمانى (تعداد) } & \multirow{2}{*}{ متثير } \\
\hline تركييى (7) (درصد) & ثبات مركزى (7) (درصد) & ثبات كُردنى (7) (درصد) & \\
\hline$r(\Delta \cdot)$ & $r(\Delta+)$ & $l(I \& / V)$ & \multirow{3}{*}{ جنسيت (تعلاد) } \\
\hline & & & \\
\hline$r(\Delta \cdot)$ & $r(\Delta \cdot)$ & $\Delta(A T / M)$ & \\
\hline$I(1 \& N)$ & $r(\Delta \cdot)$ & $r(T M / M)$ & \multirow{3}{*}{ سطح تحصيلات (تعلداد) } \\
\hline$r(\Delta \cdot)$ & $r(M T / N)$ & $1(1 \& / V)$ & \\
\hline$r(M / M / \mu)$ & $1(1 \& N)$ & $r(\Delta \cdot)$ & \\
\hline $88 / 0 \cdot \pm 1 / A \gamma$ & $\gamma \cdot / / \gamma \pm r / q$ & ENIV $\pm \& / .1$ & سن (سال) (ميانكين +انحرافمعيار) \\
\hline$r \cdot / \kappa q \pm r / ৭ q$ & $r N \cdot r \pm H / R^{H}$ & WVIRY $\pm r / r \Delta \Delta$ & شاخص توده بلنى (ميانكين +انحرافععيار) (kg/m²) \\
\hline
\end{tabular}


جدول r. مقايسه ميزان شدت درد يس از تمرينات در سه گروه درمانى

\begin{tabular}{|c|c|c|c|c|c|c|c|}
\hline Pt & 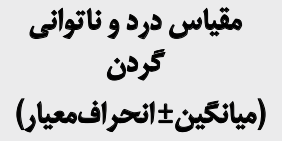 & Pt & 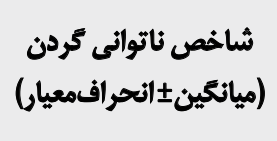 & Pt & 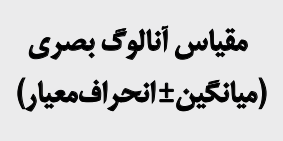 & زمان آزمون & 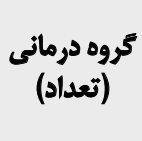 \\
\hline \multirow{3}{*}{$\circ \ldots$} & $\Delta E / P Y \pm T / I$ & & $p q / / V \pm r / N$ & & $8 / \cdot A \pm \cdot / \Delta A$ & قبل از تمرين & \multirow{3}{*}{ 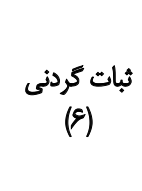 } \\
\hline & $\Delta \mathrm{H} / A \mathrm{r} \pm \mathrm{N} / \mathrm{Q}$ & $.10 . . *$ & $P V / . \pm T / Q$ &.$/ * \Lambda^{* *}$ & $\Delta / T \Delta \pm . / 8 q$ & A هفته بعد & \\
\hline & $\Delta . / \cdot r \pm$ I/Fe & & $r q / I V \pm T / N q$ & & $r / A Y \pm \cdot / \Delta Y$ & זI هفته بعد & \\
\hline \multirow{3}{*}{$+1+\cdots *$} & $\Delta F / \cdot 1 \pm T / P F$ & & $r q / \& V \pm 1 / N$ & & $9 / \cdot \pm \cdot \Delta / \Delta \Delta$ & قبل از تمرين & \multirow{3}{*}{$\begin{array}{c}\text { ثبات مركزى } \\
\text { (9) }\end{array}$} \\
\hline & $\Delta r / F F \pm . / F A$ & $.1 . . *$ & $r r / R V \pm r / A$ & $\cdot 1 \cdot \mathrm{K}^{*}$ & $\Delta / T \Delta \pm \cdot / A r$ & A هفته بعد & \\
\hline & PNAT \pm /IF & & $r q / / V \pm 1 / q \varphi$ & & $r / q r \pm . / r$. & rا هفته بعد & \\
\hline \multirow{3}{*}{$.10 . * *$} & $\Delta \Delta / Y \Delta \pm \cdot / r A$ & & $r q / A r \pm r / r r$ & & $8 / . . \pm . / F a$ & قبل ازتمرين & \multirow{3}{*}{ تركيبى } \\
\hline & $\Delta r / P V \pm \cdot / R r$ & $.1 \ldots$ & $R T / E V \pm V / N$ & $.1 \ldots \ldots$ & $\mid(q N \pm . / F)$ & A هفتهله بعد & \\
\hline & $P V / \Delta I \pm I / P f$ & & $\mathrm{r} / \mathrm{V} \pm \mathrm{r} / \mathrm{N} \varepsilon$ & & $r / * \pm * / \mu r$ & |r هفته بعد & \\
\hline
\end{tabular}

جدول ب. مقايسه ميزان شدت درد يس از تمرينات در سه كروه درمانى با درنظركرفتن اثر زمان و متغيرهاى زميئهاي

\begin{tabular}{|c|c|c|c|c|c|}
\hline $\mathbf{P}$ & $\mathbf{F}$ & درجه آزادى & مثدار اثر متغير & متغير & شاخص \\
\hline$+1+*$ & QNHA** & $r$ &.$/ 9 Y A$ & زمان & \\
\hline D מ & $f / q^{*}$ & $r$ &.$/ 909$ & كروه درمانى & \\
\hline . MEA & $1 / / r$ & $r$ & $\cdot / r+*$ & جنسيت & مقياس آنالوى \\
\hline . MIT & $1 / 10$ & $r$ & $\cdot \mid 8+1$ & سطح تحصيلات & بصرى \\
\hline $.18 \Delta \mathrm{r}$ &. $\mid P F i$ & $r$ & $.1 \cdot 19$ & سن & \\
\hline.$/ 91 \mathrm{~V}$ & $.1 . A Y$ & $r$ & .1 .19 & شاخص توده بلدنى & \\
\hline .10 & IPV/TE** & $r$ &.$/ 9 Y$ & زمان & \\
\hline 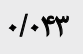 & $p / 4 q q^{*}$ & r & . /AVA & كروه درمائى & \\
\hline H.r & $9 / \pi I^{\circ}$ & r & . Mr & جنسيت & شاخص ناتوائي \\
\hline.$/ M I I$ & $1 / 81$ & p & . /PAR & سطح تحصيلات & كردن \\
\hline ./NP & $r / P Y$ & r & . rrar & سن & \\
\hline $.1 \times 1$ & $r / A T$ & $r$ & . & شاخص توده بدنى & \\
\hline $.1+$ & $V \in / \pi q * *$ & $r$ & . & زمان & \\
\hline.$/ .4 q$ & $f / \Delta C^{*}$ & $r$ &.$/ 911$ & كروه درمانى & \\
\hline - IEAY &.$/ 4 t$ & r & $1 / 19$ & جنسيت & مقياس درد و \\
\hline .1819 &.$\angle A E A$ & r & $. / 4+F$ & سطح تحصيلات & ناتوانى كردن \\
\hline.$/ 019$ & $\cdot N \cdot V$ & r & ع & سن & \\
\hline .1811 & $\cdot / \Delta \cdot V$ & $r$ &.$/ 1.1$ & شاخص توده بديثي & \\
\hline
\end{tabular}




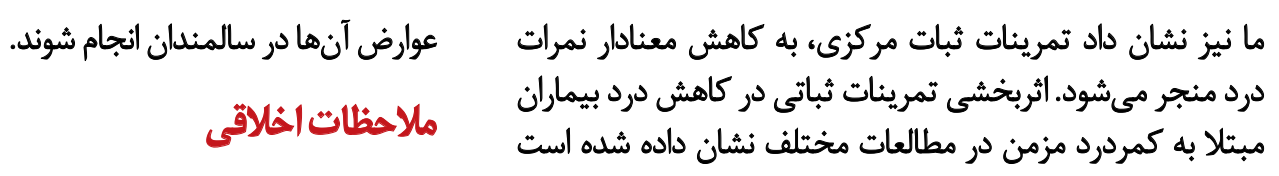

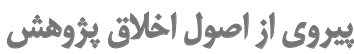

اين مطالعه در مركز ثبت كارآزمايى بالينى ايران با كد ثبت

(IRCT20180412039280N2)

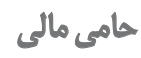

اين مطالعه تحت حمايت مالى مركز تحقيقات سالمندى دانشكاه

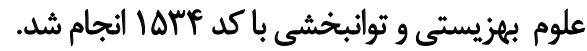

$$
\text { مشاركت نويسندكَان }
$$

تمام نويسندكان در طراحى، اجرا و نعارش همه بخشهاي يُؤوهش حاضر مشاركت داشتهاند.

$$
\text { تعارض مثاقع }
$$

بنا به اظهار نويسندكان، در اين مقاله هيجّونه تعارض منافعى

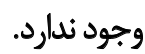

$$
\text { تشكر و قدرداثي }
$$

مجريان اين طرح از همه شركت كنندكان در يُؤهش و نيز مركز

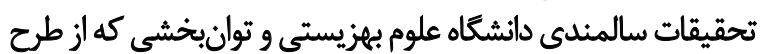
حمايت مالى كردند، تقدير و تشكر مئي كنيند.

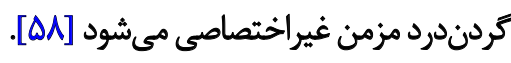

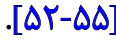

مطالعات الباندراوى و و دوسانسلى ع نشان دادند تمرينات ثباتى

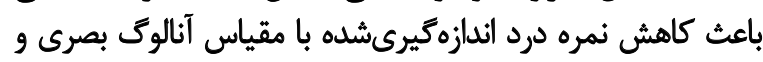

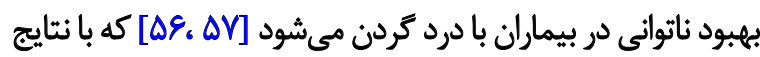

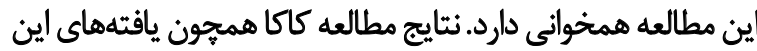

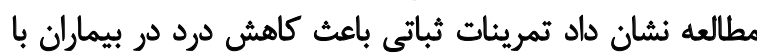

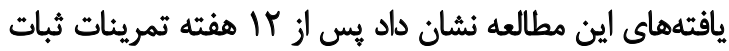

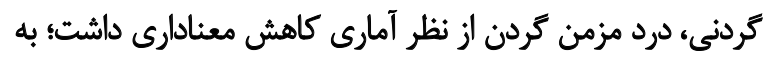

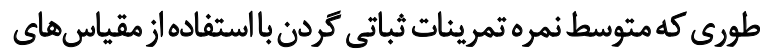

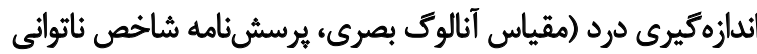

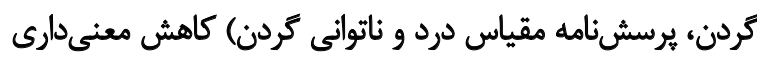

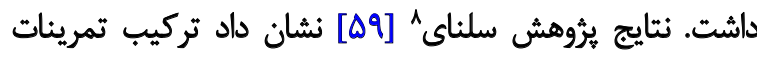

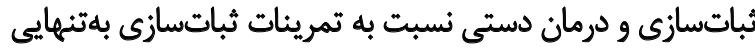

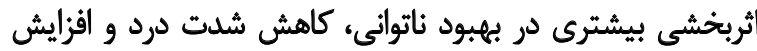

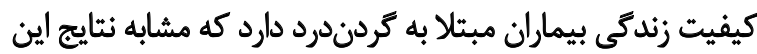

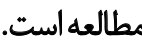

\section{تثيجه تيرى نمايى}

يافتههاي اين مطالعه نشان داد تمرينات ثبات كردنى، مركزى و

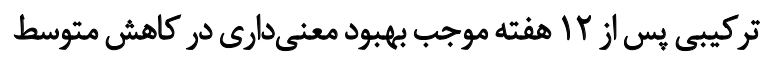

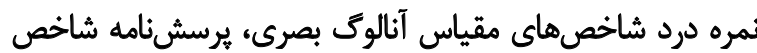

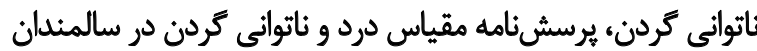

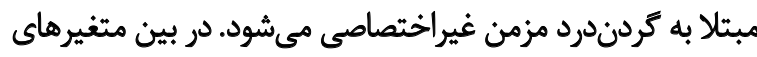

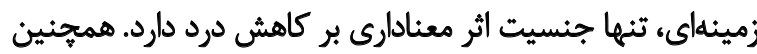

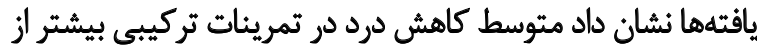

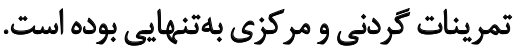

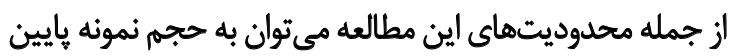

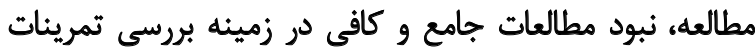

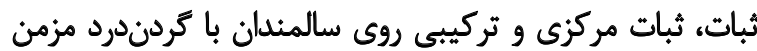

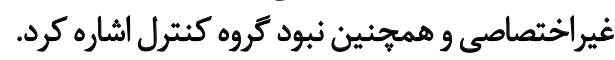

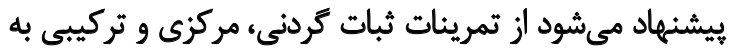

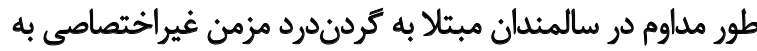

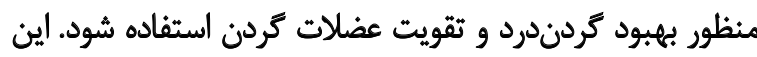

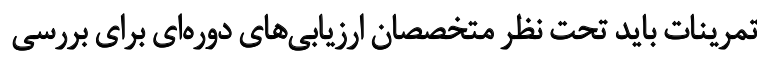
اختلالات كردندرد و شناسايى بلهموقع اين اختلالات و درمائ بران برسي 


\section{References}

[1] Parno A, Sayehmiri K, Mokarami H, Parno M, Azrah K, Ebrahimi MH, et al. [The prevalence of work-related musculoskeletal disorders in the lower limbs among Iranian workers: A meta-analysis study (Persian)]. Iran Occupational Health. 2016; 13(5):50-9.

[2] Khandan M, Koohpaei A, Kohansal Aghchay M, Ebrahimi M H, Khammar A, Arsang Jang S, et al. Assessing the factors predicting work-related musculoskeletal disorders among Iranian port's personnel using regression model. Iranian Rehabilitation Journal. 2017; 15(4):309-16. [DOI:10.29252/nrip.irj.15.4.309]

[3] Khandan M, Ataei manesh L, Eyni Z, Khosravi Z, Biglari H, Koohpaei AR, et al. [Relationship between Job content and demographic variables with musculoskeletal disorders among nurses in a university hospital, Qom Province, 2014 (Persian)]. Research Journal of Applied Sciences. 2016; 11(7):547-53.

[4] Parno A, Sayehmiri K, Parno M, Khandan M, Poursadeghiyan $\mathrm{M}$, Maghsoudipour M, et al. The prevalence of occupational musculoskeletal disorders in Iran: A meta-analysis study. Work. 2017; 58(2):203-14. [DOI:10.3233/WOR-172619] [PMID]

[5] Ylinen J, Takala EP, Nykänen M, Häkkinen A, Mälkiä E, Pohjolainen $\mathrm{T}$, et al. Active neck muscle training in the treatment of chronic neck pain in women: A randomized controlled trial. JAMA. 2003; 289(19):2509-16. [DOI:10.1001/jama.289.19.2509] [PMID]

[6] Hush JM, Maher CG, Refshauge KM. Risk factors for neck pain in office workers: A prospective study. BMC Musculoskeletal Disorders. 2006; 7:81. [DOI:10.1186/1471-2474-7-81] [PMID] [PMCID]

[7] Koohpaei A, Khandan M, Vosoughi S, Khammar A, Mobinizade V, Farrokhi M, et al. Industrial workers' postures analysis by a new method named "loading on the upper body assessment" in Iran. Annals of Tropical Medicine and Public Health. 2017; 10(4):973-7. [DOI:10.4103/ATMPH.ATMPH_304_17]

[8] Rajabi R, Minoonejad H, Karimi-Zadeh Ardakani M, Darzi Sheikh Z, Ramezani-Ouzineh M. [The relationship between Craniovertebral (CV) Angle and neck pain among male and female students with an emphasis on different educational levels (Persian)]. Journal of Rehabilitation. 2015; 16(3):218-27.

[9] Hush JM, Michaleff Z, Maher CG, Refshauge K. Individual, physical and psychological risk factors for neck pain in Australian office workers: A 1-year longitudinal study. European Spine Journal. 2009; 18(10):1532-40. [DOI:10.1007/s00586-009-1011-z] [PMID] [PMCID]

[10] Carroll L. The factors associated with neck pain and its related disability in the Saskatchewan population. Spine. 2000; 25(9):110917. [DOI:10.1097/00007632-200005010-00012] [PMID]

[11] McNair PJ, Portero P, Chiquet C, Mawston G, Lavaste F. Acute neck pain: Cervical spine range of motion and position sense prior to and after joint mobilization. Manual Therapy. 2007; 12(4):390-4 [DOI:10.1016/j.math.2006.08.002] [PMID]

[12] Kim NJ, Kim JS, Wang JS, Park JH, Choi JH. The effects of isometric trunk exercises and dynamic trunk exercises on gait in elderly people. Journal of Physical Therapy Science. 2015; 27(6):1685-9. [DOI:10.1589/jpts.27.1685] [PMID] [PMCID]

[13] Häkkinen A, Kautiainen H, Hannonen P, Ylinen J. Strength training and stretching versus stretching only in the treatment of patients with chronic neck pain: A randomized one-year follow-up study. Clinical Rehabilitation. 2008; 22(7):592-600. [DOI:10.1177/0269215507087486] [PMID]
[14] Salo PK, Häkkinen AH, Kautiainen H, Ylinen JJ. Effect of neck strength training on health-related quality of life in females with chronic neck pain: A randomized controlled 1-year follow-up study. Health and Quality of Life Outcomes. 2010; 8:48. [DOI:10.1186/1477-7525-8-48] [PMID] [PMCID]

[15] Rezasoltani A, Khaleghifar M, Tavakoli A, Ahmadipour A. [The comparison of neuromuscular facilitation exercises and traditional exercise therapy programs in the treating of patients with chronic non-specific neck pain (Persian)]. Journal of Rafsanjan University of Medical Sciences. 2009; 8(1):59-68.

[16] McLean SM, May S, Klaber-Moffett J, Sharp DM, Gardiner E. Risk factors for the onset of non-specific neck pain: A systematic review. Journal of Epidemiology \& Community Health. 2010; 64(7):565-72. [DOI:10.1136/jech.2009.090720] [PMID]

[17] Malek M, Hassankhani H, Darvishpur Kakhki A, Asghari Jafarabadi M, Mansouri Arani M, Mohammad Nejad. [Investigating of quality of life and it's correlation with individual, social, economic, and health variables of retirees of Tabriz (Persian)]. Iranian Journal of Nursing Research. 2016; 11(1):34-41.

[18] Kun LG. Telehealth and the global health network in the 21st century. From homecare to public health informatics. Computer Methods and Programs in Biomedicine. 2001; 64(3):155-67. [DOI:10.1016/S0169-2607(00)00135-8]

[19] Viljanen M, Malmivaara A, Uitti J, Rinne M, Palmroos P, Laippala P. Effectiveness of dynamic muscle training, relaxation training, or ordinary activity for chronic neck pain: Randomised controlled trial. BMJ. 2003; 327(7413):475. [DOI:10.1136/ bmj.327.7413.475] [PMID] [PMCID]

[20] Fejer R, Kyvik KO, Hartvigsen J. The prevalence of neck pain in the world population: A systematic critical review of the literature. European Spine Journal. 2006; 15(6):834-48. [DOI:10.1007/ s00586-004-0864-4] [PMID] [PMCID]

[21] Stranjalis G, Kalamatianos T, Stavrinou LC, Tsamandouraki K, Alamanos Y. Neck pain in a sample of Greek urban population (fifteen to sixty-five years): Analysis according to personal and socioeconomic characteristics. Spine. 2011; 36(16):1098-104. [DOI:10.1097/BRS.0b013e3182054add] [PMID]

[22] Hoy D, Protani M, De R, Buchbinder R. The epidemiology of neck pain. Best Practice \& Research Clinical Rheumatology. 2010; 24(6):783-92. [DOI:10.1016/j.berh.2010.10.002] [PMID]

[23] Douglass AB, Bope ET. Evaluation and treatment of posterior neck pain in family practice. The Journal of the American Board of Family Practice. 2004; 17(suppl 1):S13-S22. [DOI:10.3122/jabfm.17.suppl_1.S13] [PMID]

[24] Rao R. Neck pain, cervical radiculopathy, and cervical myelopathy: Pathophysiology, natural history, and clinical evaluation. Journal of Bone and Joint Surgery. 2002; 84(10):1872-81. [PMID]

[25] Young WF. Cervical spondylotic myelopathy: A common cause of spinal cord dysfunction in older persons. American Family Physician. 2000; 62(5):1064-70. [PMID]

[26] Taimela S, Takala E-P, Asklöf T, Seppälä K, Parviainen S. Active treatment of chronic neck pain: A prospective randomized intervention. Spine. 2000; 25(8):1021-7. [DOI:10.1097/00007632200004150-00018] [PMID]

[27] Cote P, Cassidy JD, Carroll L. The factors associated with neck pain and its related disability in the Saskatchewan population. Spine. 2000; 25(9):1109-17. [DOI:10.1097/00007632-20000501000012] 
[28] Kay TM, Gross A, Goldsmith C, Santaguida PL, Hoving J, Bronfort G. Exercises for mechanical neck disorders. The Cochrane Database of Systematic Reviews. 2005; 20(3):CD004250. [DOI:10.1002/14651858.CD004250.pub3]

[29] Andersson HI, Ejlertsson G, Leden I, Scherstén B. Musculoskeletal chronic pain in general practice: Studies of health care utilisation in comparison with pain prevalence. Scandinavian Journal of Primary Health Care. 1999; 17(2):87-92. [DOI:10.1080/0281343997 50002700] [PMID]

[30] Clark M, Fater D, Reuteman P. Core (trunk) stabilization and its importance for closed kinetic chain rehabilitation. Orthopaedic Physical Therapy Clinics of North America. 2000; 9(2):119-36.

[31] Akbari A, Ghiasi F, Barahoie M, Arab-Kangan M. [The comparison of effectiveness of muscles specific stabilization training and dynamic exercises on the chronic neck pain and disability (Persian)]. Journal of Gorgan University of Medical Sciences. 2010; 11(4):29-112.

[32] Ilbeigi S, Nikbin L, Afzalpour ME. [The effect of six weeks of core stability exercise on pain and trunk muscle endurance in girl students with chronic non-specific low back pain (Persian)]. Journal of Torbat Heydariyeh University of Medical Sciences. 2014; 2(2):5-13.

[33] Safdari S, Khayambashi K, Ghasemi GA, Falah A, Sakhavat E. [Effects of selected core stabilization exercise protocol on pain and functional disability in subjects with chronic non-specific low back pain (Persian)]. Journal of Research in Rehabilitation Sciences. 2014; 10(1):56-66.

[34] Drescher K, Hardy S, MacLean J, Schindler M, Scott K, Harris SR. Efficacy of postural and neck-stabilization exercises for persons with acute whiplash-associated disorders: A systematic review. Physiotherapy Canada. 2008; 60(3):215-23. [DOI:10.3138/ physio.60.3.215] [PMID] [PMCID]

[35] Noh HJ, Shim JH, Jeon YJ. [Effects of neck stabilization exercises on neck and shoulder muscle activation in adults with forward head posture (Persian)]. International Journal of Digital Content Technology and its Applications. 2013; 7(12):492-8.

[36] Ahn JA, Kim JH, Bendik AL, Shin JY. Effects of stabilization exercises with a Swiss ball on neck-shoulder pain and mobility of adults with prolonged exposure to VDTs. Journal of Physical Therapy Science. 2015; 27(4):981-4. [DOI:10.1589/jpts.27.981] [PMID] [PMCID]

[37] Kisner C, Colby LA. Therapeutic exercise: Foundations and techniques. Philadelphia: Fa Davis; 2012

[38] Asgari-Ashtiani AR, Ebrahimi-Takmajani E, Torkaman G, Amiri M, Mohammadi M. [The effects of stabilization exercises and maximum isometric exercises on fear avoidance of belief in chronic non-specific neck pain (Persian)]. Archives of Rehabilitation. $2014 ; 15(2): 22-31$.

[39] Bolandian P, Rajabi R, Alizadeh M, Sohrabi A, Miri H, Bolandian A. [Efficacy of eight week of exercise therapy for neck pain among general dentists (Persian)]. Journal of Islamic Dental Association of Iran. 2015; 27(2):104-8.

[40] Taheri H. [The evaluation of the effect of therapeutic exercises programs on the rate of chronic neck pain and disability in police staff of Lorestan (Persian)]. Police Medicine. 2014; 3(3):193-202.

[41] Arami J, Rezasoltani A, Khalkhali Zaavieh M, Rahnama L. [The effect of two exercise therapy programs (proprioceptive and endurance training) to treat patients with chronic non-specific neck pain (Persian)]. Journal of Babol University of Medical Sciences. 2012; 14(1):77-84.

[42] Letafatkar A, Taghavi M, Alizadeh MH, Hadadnezhad M, Norouzi H. [The effect of one period of exercise program on non athlete's neck rom and chronic neck pain (Persian)]. Journal of Research in Rehabilitation Sciences. 2011; 7(1):39-47.

[43] Hemmati S, Rajabi R, Karimi N. [Effects of consecutive supervised core stability training on pain and disability in women with nonspecific chronic low back pain (Persian)]. Koomesh. 2011; 12(3):244-52.

[44] Nezhad Roomezi S, Rahnama N, Habibi A, Negahban H. [The effect of core stability training on pain and performance in women patients with non-specific chronic low back pain (Persian)] Journal of Rehabilitation Sciences and Research. 2012; 8(1):57-64.

[45] Ahmed R, Shakil-ur-Rehman S, Sibtain F. Comparison between specific lumber mobilization and core-stability exercises with core-stability exercises alone in mechanical low back pain. Pakistan Journal of Medical Sciences. 2014; 30(1):157-60. [DOI:10.12669/pjms.301.4424] [PMID] [PMCID]

[46] Ebrahimi H, Blaouchi R, Eslami R, Shahrokhi M. Effect of 8-week core stabilization exercises on low back pain, abdominal and back muscle endurance in patients with chronic low back pain due to disc herniation. Physical Treatments - Specific Physical Therapy. 2014; 4(1):25-32.

[47] Hajihasani AH, Hedayati R, Ehsani F. [Effect of core stability and general exercise on functional activity in non-specific low back pain patients (Persian)]. Journal of Gorgan University of Medical Sciences. 2016; 18(2):13-8

[48] Karimzadeh F, Letafatkar A, Ghasemi G. [The effect of 8 weeks core stabilization exercises on pain and functional disability induced by low back pain in the mothers of children with cerebral palsy (Persian)]. Scientific Journal of Kurdistan University of Medical Sciences. 2016; 21(3):34-44.

[49] Rastegar M, Haghighi A, Askari R. [Effect of core stabilization exercise on the reduction of low back pain and ultrasonic changes of multifidus in aged-women with chronic low back pain (Persian)]. Anesthesiology and Pain. 2016; 6(4):62-74

[50] Yalfani A, Ahmadnezhad L, Gholami Borujeni B, Khoshnamvand $Z$. The Effect of six weeks core stability exercise training on balance, pain and function in women with chronic low back pain Journal of Health and Care. 2017; 18(4):336-46.

[51] Sartipzadeh M, Moazami M, Mohammadi M. [The effect of core stabilization training on elderly balance and knee pain with knee osteoarthritis (Persian)]. Journal of Paramedical Sciences \& Rehabilitation. 2016; 5(3):7-17.

[52] Lee TR, Kim YH, Sung PS. A comparison of pain level and entropy changes following core stability exercise intervention Medical Science Monitor. 2011; 17(7):CR362-8. [DOI:10.12659/ MSM.881846] [PMID] [PMCID]

[53] Ezati KN, Talimkhani I, Esmaeili K, Sherafat SH. [The effects of supervised core stability training on clinical symptoms, range of motion, and endurance of lumbar muscles in female patients with non-specific chronic low back pain (Persian)]. Physical Treatments-Specific Physical Therapy. 2012; 2(1):23-30.

[54] Motallebi L, Mohseni Bandpei MA, Rahmani N. [Effects of stabilization exercises on pain intensity, functional disability and cross sectional area of multifidus muscle in women with non- 
specific chronic low back pain (Persian)]. Journal of Mazandaran University of Medical Sciences. 2013; 23(100):11-9.

[55] Shakeri A SS, Sokhangoei Y, Soseini Y. [Comparison of three methods of massage therapy, stability exercise and combination exercise on pain and disability in patients with nonspecific chronic low back pain (Persian)]. Anesthesiology and Pain. 2015; 5(2):55-62.

[56] El-Bandrawy AM, Ghareeb HO. Efficacy of neck stabilization exercises for neck pain complicating pregnancy. International Journal of Therapies and Rehabilitation Research. 2016; 5(5):1228. [DOI:10.5455/ijtrr.000000193]

[57] Dusunceli Y, Ozturk C, Atamaz F, Hepguler S, Durmaz B. Efficacy of neck stabilization exercises for neck pain: A randomized controlled study. Journal of Rehabilitation Medicine. 2009; 41(8):626-31. [DOI:10.2340/16501977-0392] [PMID]

[58] Kaka B, Ogwumike O, Adeniyi F. Efficacy of neck stabilisation and dynamic exercises on pain intensity, depression and anxiety among patients with non-specific neck pain. Physiotherapy. 2016; 102s(2016):es1-42. [DOI:10.1016/j.physio.2016.10.005]

[59] Celenay ST, Akbayrak T, Kaya DO. A comparison of the effects of stabilization exercises plus manual therapy to those of stabilization exercises alone in patients with nonspecific mechanical neck pain: A randomized clinical trial. Journal of Orthopaedic \& Sports Physical Therapy. 2016; 46(2):44-55. [DOI:10.2519/ jospt.2016.5979] [PMID] 